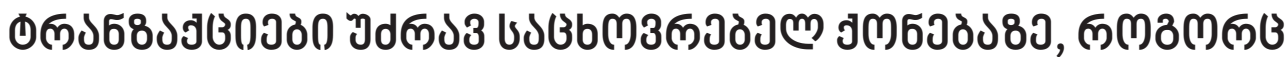

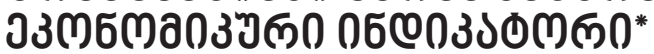

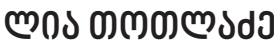

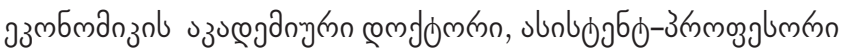

https://doi.org/10.35945/gb.2018.06.031

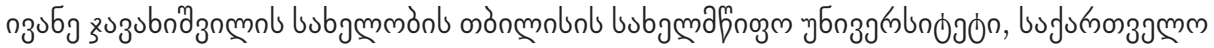

Lia.totladze@tsu.ge

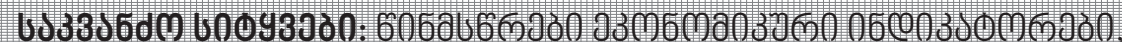

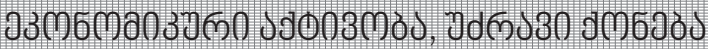

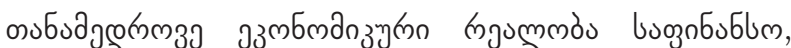

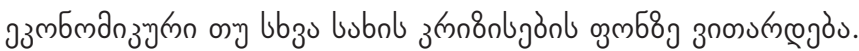

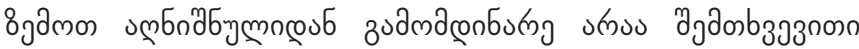

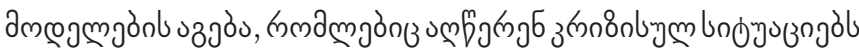

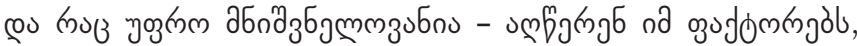

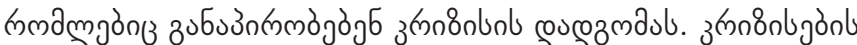

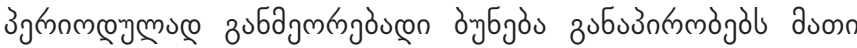

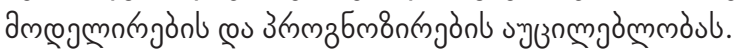

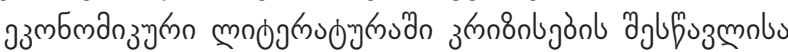

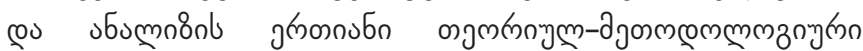

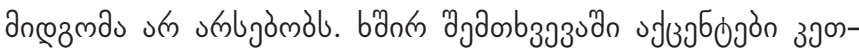

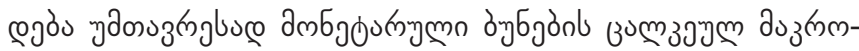

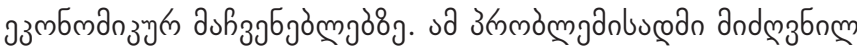

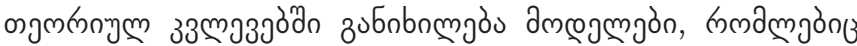

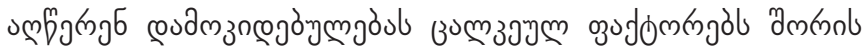

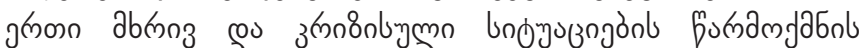

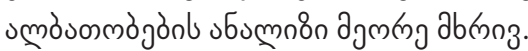

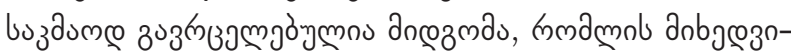

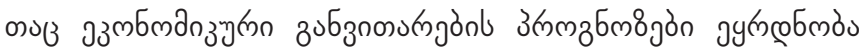

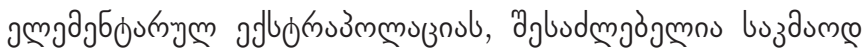

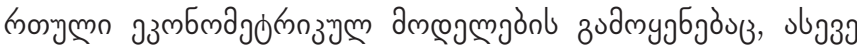

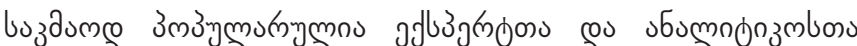

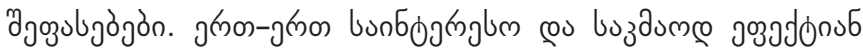

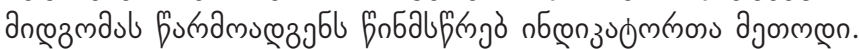
пз muonzol r mmzm m

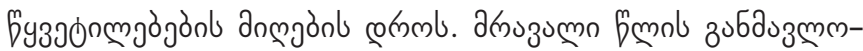

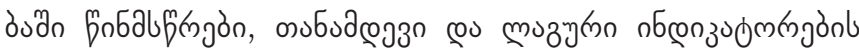

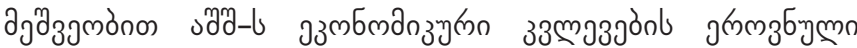

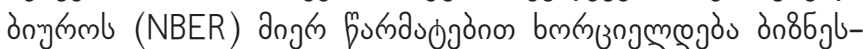

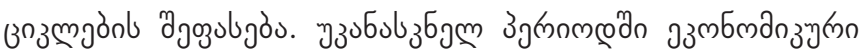

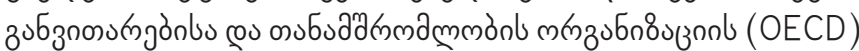

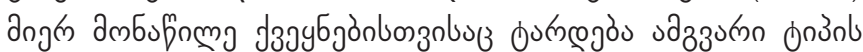

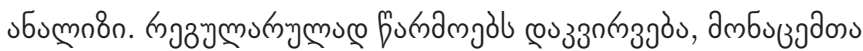

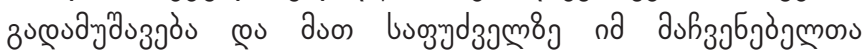

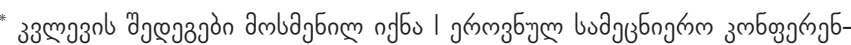

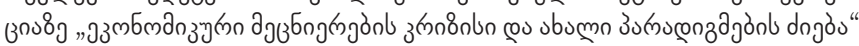

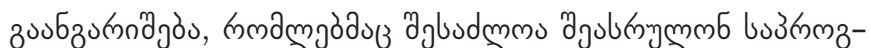

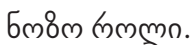

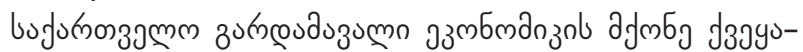

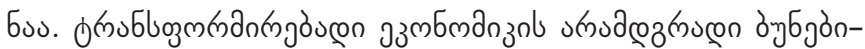

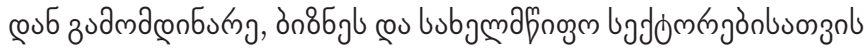

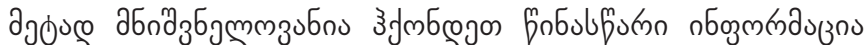

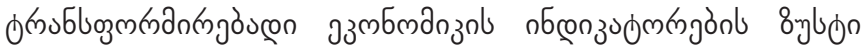

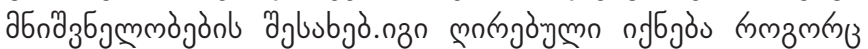

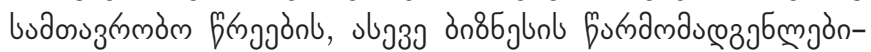

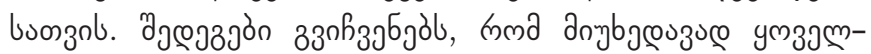

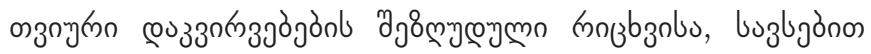

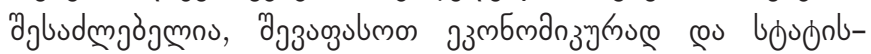

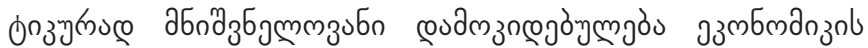

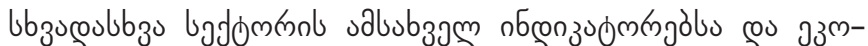

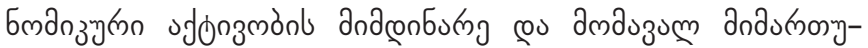

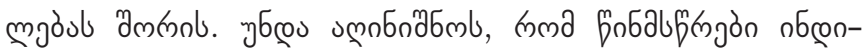

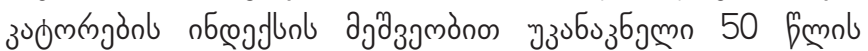

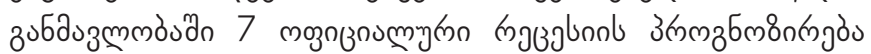

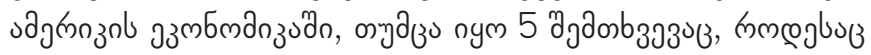

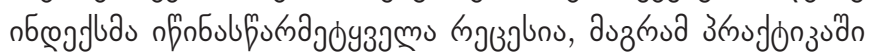
joا o or amboos.

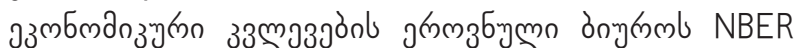

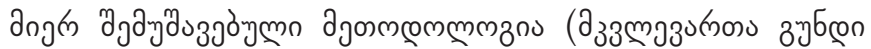

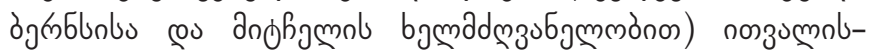

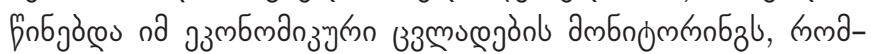

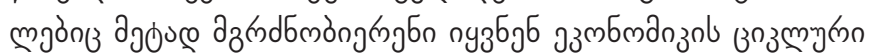
उзmnmajàjönusodn.

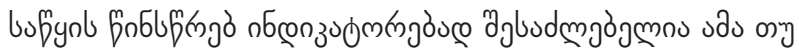

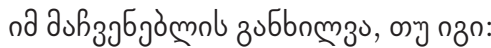

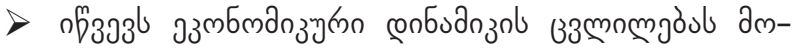

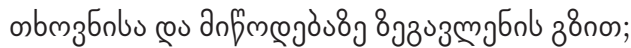

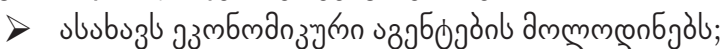

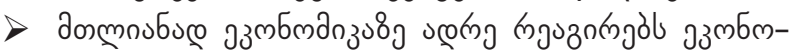

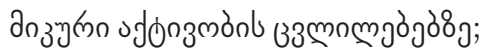

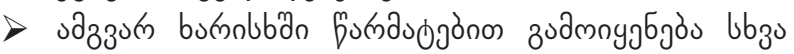

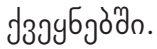

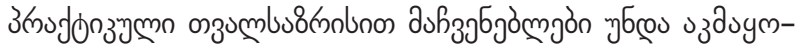

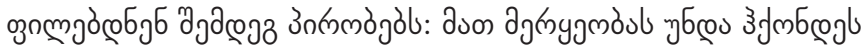




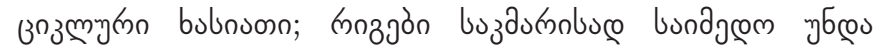

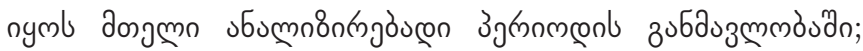

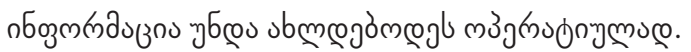

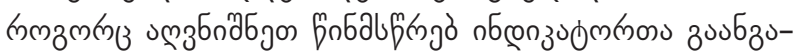

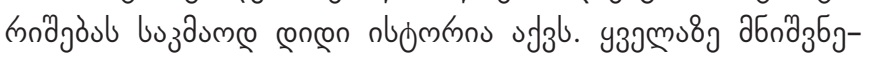

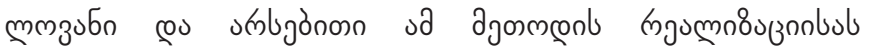

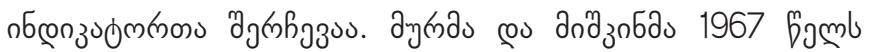

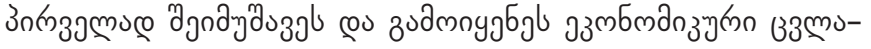

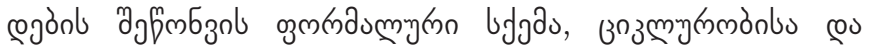

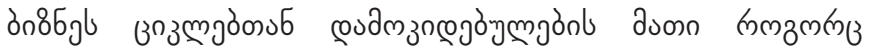

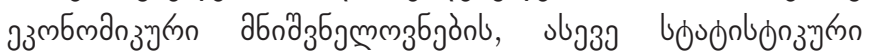

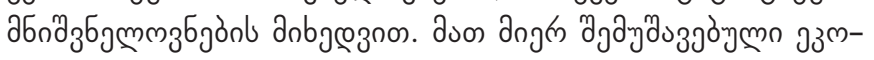

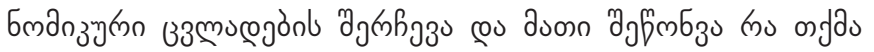

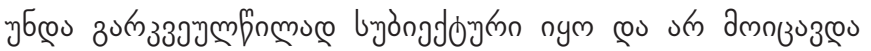

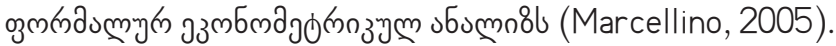

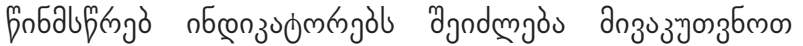

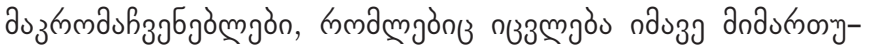

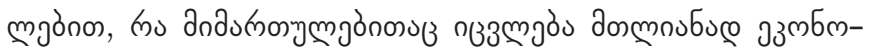

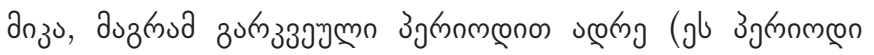

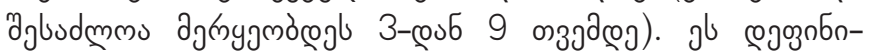

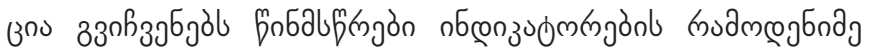

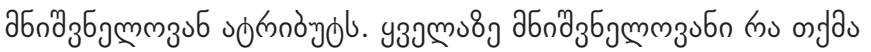

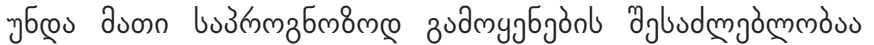
(Composite Leading Indicators ...2006).

NBER-nl agonmenmmannl mububauce zublu8

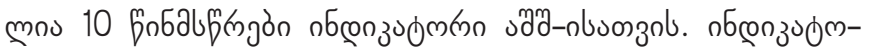

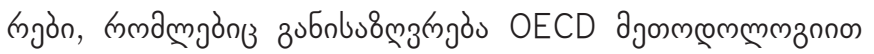

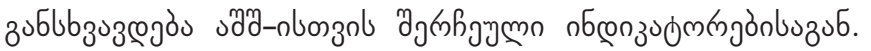

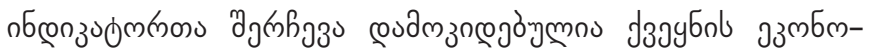

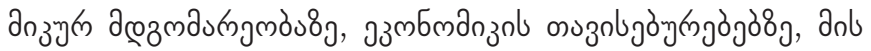

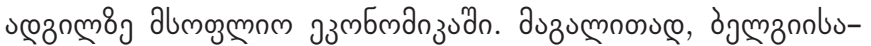
कзूl zublus

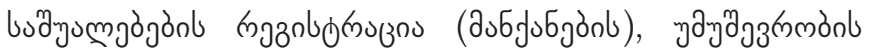

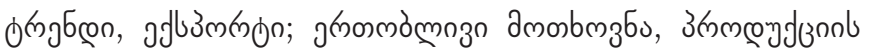

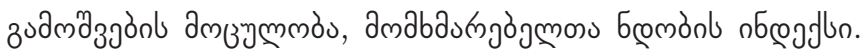

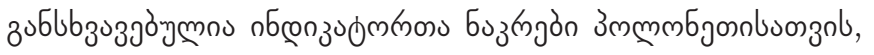

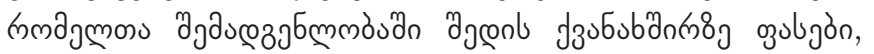

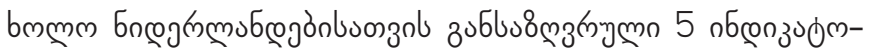

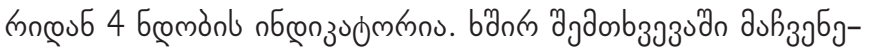

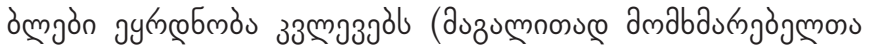

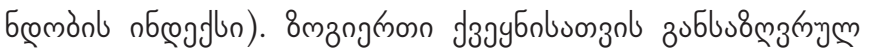

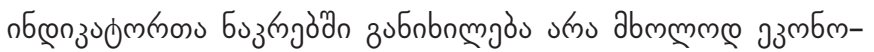

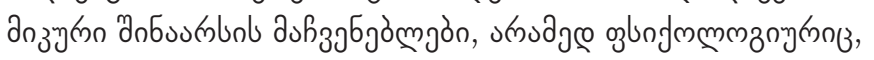

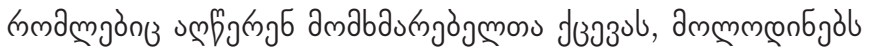
sos 0.9 .

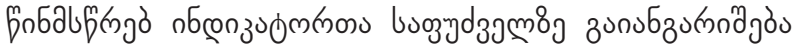

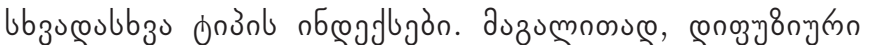

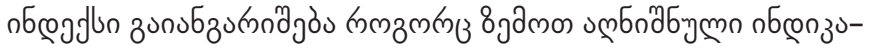

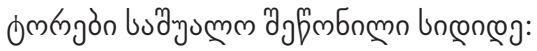

$$
L E I=\sum_{i=1}^{n} W_{i} \cdot L_{i}
$$

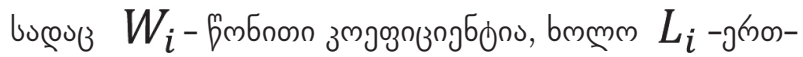

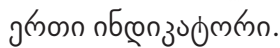

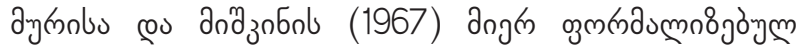

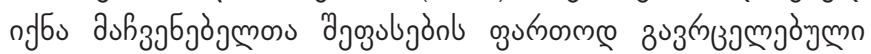

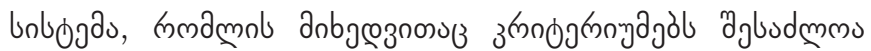

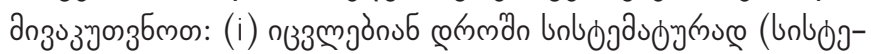

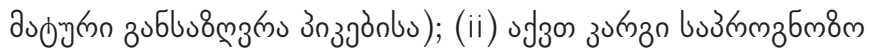

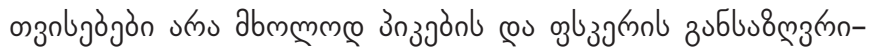

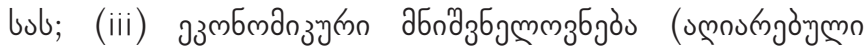

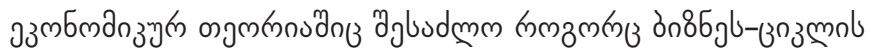

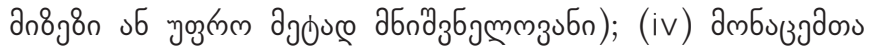

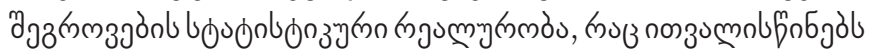

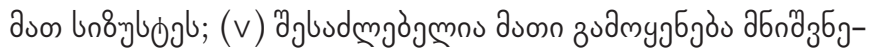

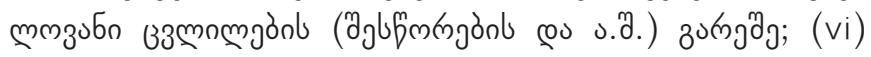

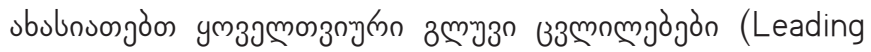
Economic Indicator, 2007).

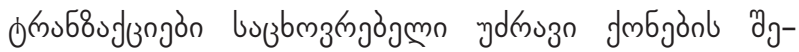

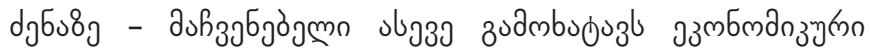

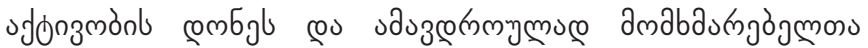

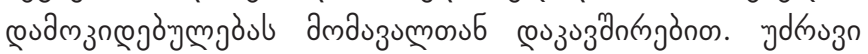

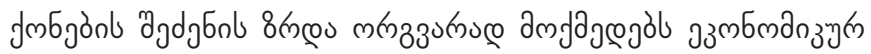

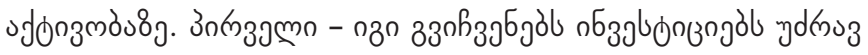

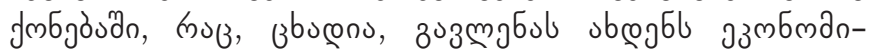

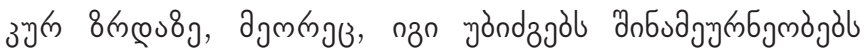

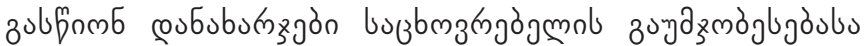
co дmৎg

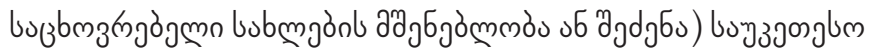

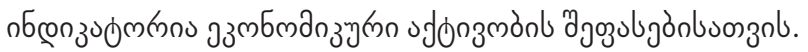

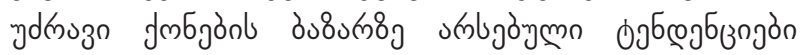

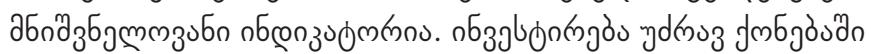

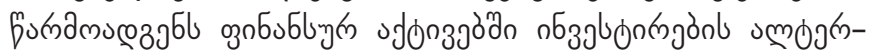
Eufnzul. ok

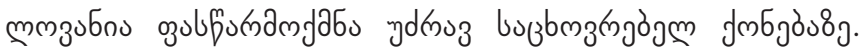

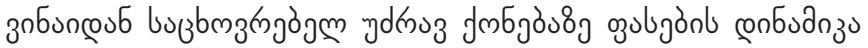

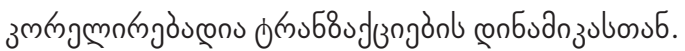

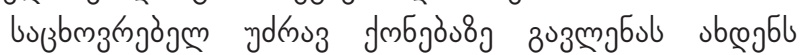

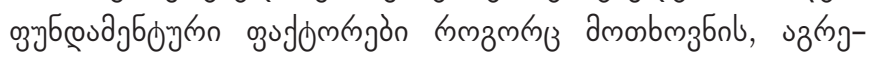

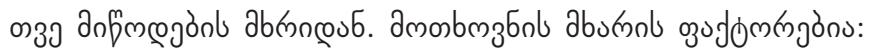

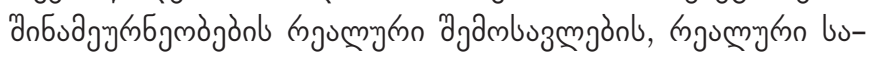

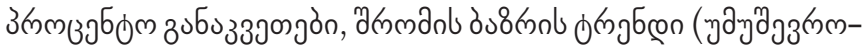

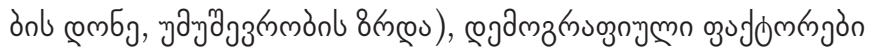

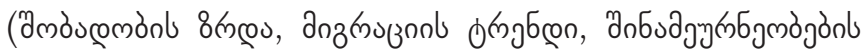

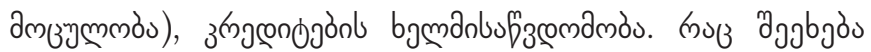

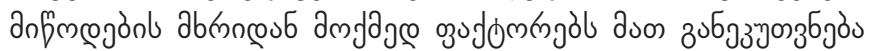




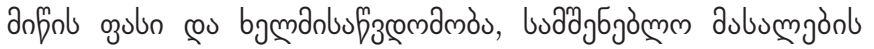

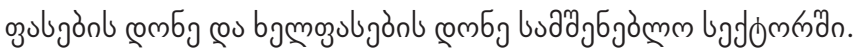

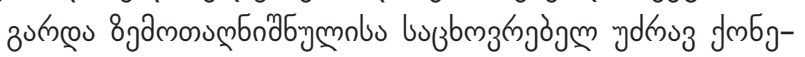

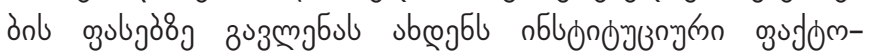

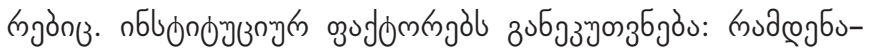

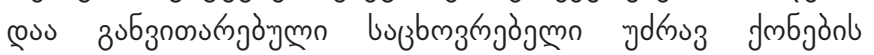

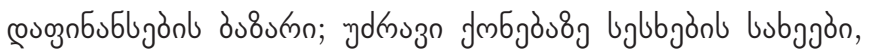

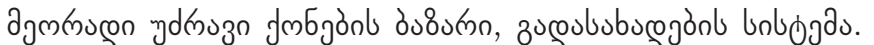

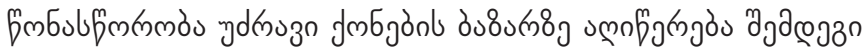
зงбommgònon:

$$
P^{H}=f\left(\stackrel{+}{Y}, \stackrel{+}{r}, \stackrel{+}{W E}, \stackrel{+}{D}, e, \bar{X}, C\left(P^{L}, \stackrel{+}{W}, M\right)\right)
$$

bocoos,

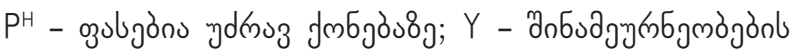

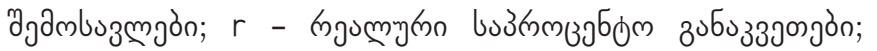

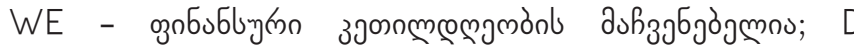

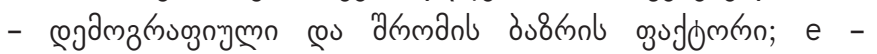

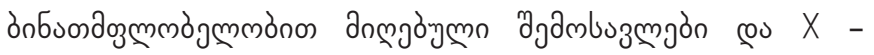

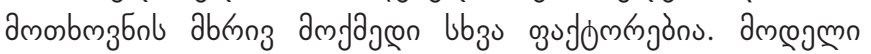

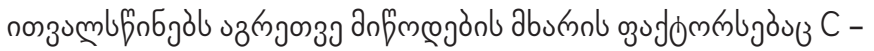

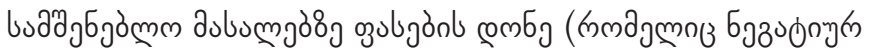

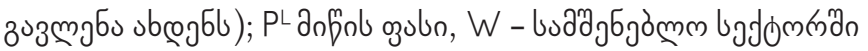
coubufajóymons bymojulo cas $M$ - coububumzgòn.

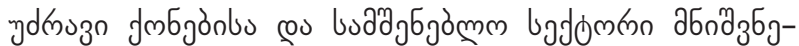

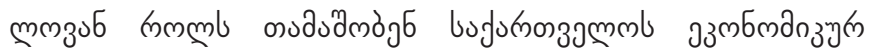
8m

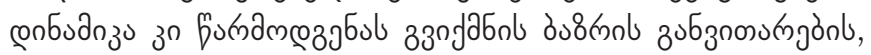

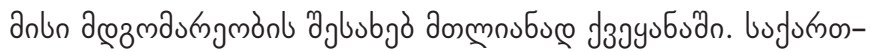

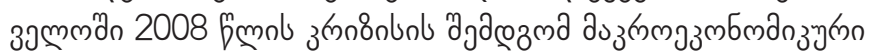

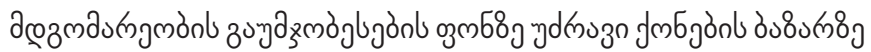

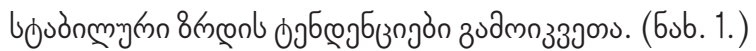

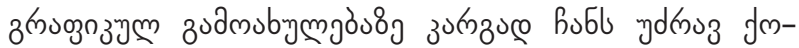

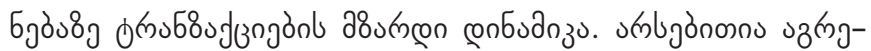

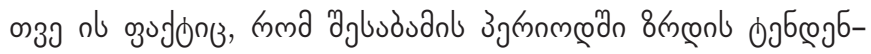

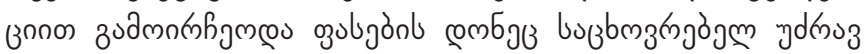

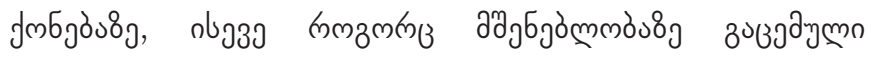

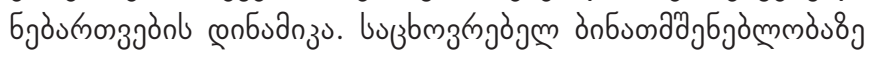

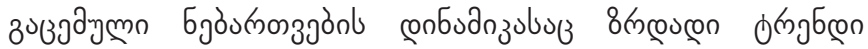

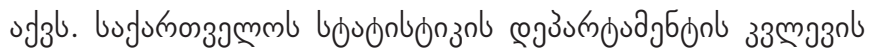

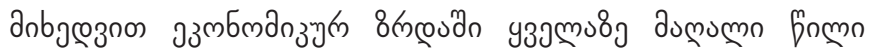

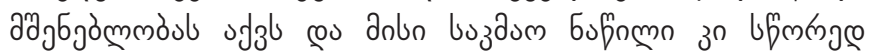

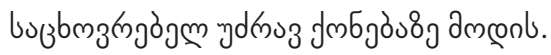

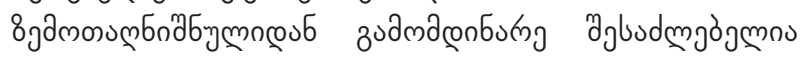
zonjzuon, nma buosubucom bojodnbonz

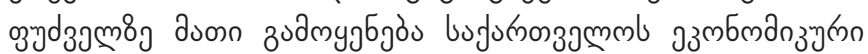

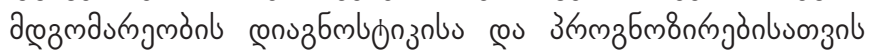

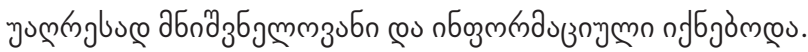

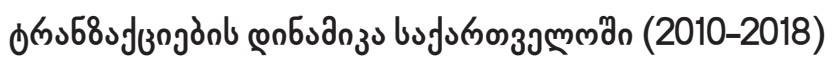

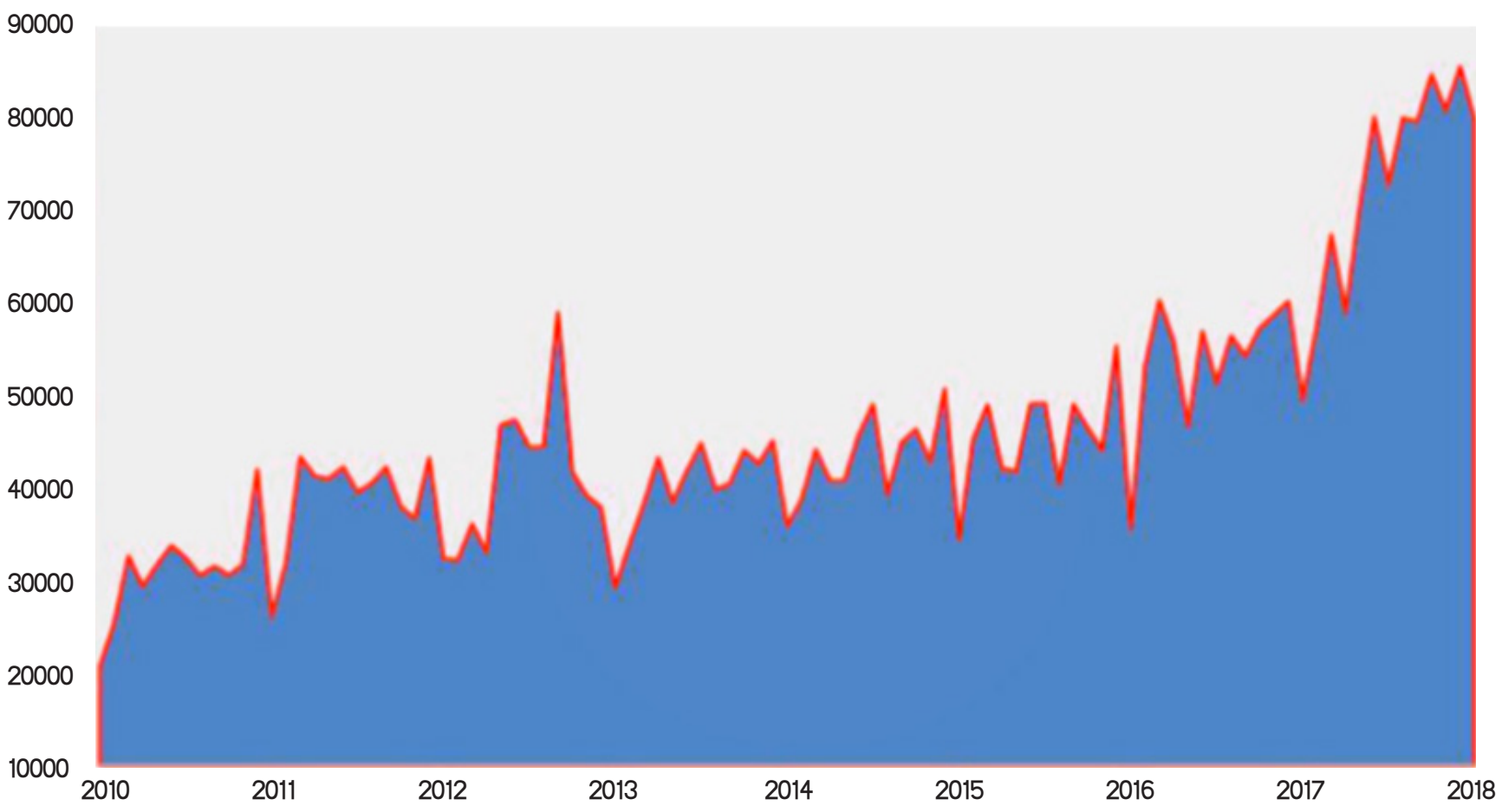




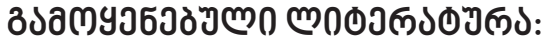

1. Leading Economic Indicator. (2007). New approaches and forecasting record. Edited by KajahLahiri and Geoffrey H. Moore. Cambridge University Press;

2. Marcellino M. (2005). Leading Indicators. IEP-Bocconi University, IGIER and CEPR;

3. Sorensen P. B. \&Whitta-Marcellino M. (2005 Jacobsen H. J. (2013), Introducing Advanced Macroeconomics: Growth and Business Cycles, 2th edition, Mc Graw-Hill;

4. Composite Leading Indicators for Major OECD Non-Member Economies and Recently New OECD Member Countries (2006). Short-term Economic Statistics Division Statistics Directorate OECD. Available at http://www.oecd.org/std/leading-indicators/36414874.pdf;

5. Estrela A., Trubin Mary R. (2006). The Yield Curve as a Leading Indicator: Some Practical Issues. Federal Reserve Bank of New York. Current Issues in Economics and Finance. Vol.12, №5;

6. Estrella, Arturo. "Why Does the Yield Curve Predict Output and Inflation?". Economic Journal, July 2005, 115 (505);

7. Totladze L. (2016). Diffusion Index for Georgia: Selection Leading Economic Indicators. International Reviewed Scientific-Analytical Journal "Economisti", №2;

8. Totladze L. (2017). DIFFUSION INDEX BASED ON LEADING ECONOMIC INDICATORS AS FORECASTING INSTRUMENT (IN CASE GEORGIA). The Geneva International Conference on Advance Research (GICAR-2107);

9. Turley ByGerard, Peter J. Luke. (2012). Transition Economics: Two Decades On. Taylor and Francis Group. London. 


\section{RESIDENTIAL TRANSACTIONS AS LEADING ECONOMIC INDICATOR}

\section{LIA TOTLADZE}

https://doi.org/10.35945/gb.2018.06.031

Academic Doctor of Economics, Assistant Professor

Ivane Javakhishvili Tbilisi State University, Georgia

Lia.totladze@tsu.ge

KEY WORLDS: LEAdING ECONOMICINDICATORS, ECONOMIC ACTIVITV, RESIDENTIAL TRANSACTION

\section{SUMMARY}

It is important to predict trends of economic development for any country. Researchers and practitioners use different ways for evaluation and forecasting economic activity. Identification of indicators, which change impact on the economy in general, is one of the widespread methods. The most appropriate tools to solve this problem are the leading indicators and indexes based on leading indicators. The selection of indicators depends on the specificity of the country's economy. Among the leading economic indicators is the dynamics of applications for the Building permissions for private houses, and can also be successfully use residential transactions. Depending on the above, the paper deals the aspects of calculation of leading economic indicators. This paper analyses some aspects of the effectiveness of indicator for predicting economic activity and describes the methodological issues forward leading indicators. Particular attention is paid to analysis of residential transactions dynamics as a leading indicator as in theoretical as in practical terms. The article highlights the peculiarities of its implementation in Georgia. 